\title{
Socio-demographic characteristics associated with emotional and social loneliness among older adults
}

Irene N. Fierloos ${ }^{1}$, Siok Swan Tan ${ }^{1}$, Greg Williams², Tamara Alhambra-Borrás ${ }^{3}$, Elin Koppelaar ${ }^{4}$, Lovorka Bilajac ${ }^{5}$, Arpana Verma ${ }^{2}$, Athina Markaki ${ }^{6}$, Francesco Mattace-Raso ${ }^{7}$, Vanja Vasiljev ${ }^{5}$, Carmen B. Franse ${ }^{1}$ and Hein Raat ${ }^{1 *}$ (D)

\begin{abstract}
Background: International studies provide an overview of socio-demographic characteristics associated with loneliness among older adults, but few studies distinguished between emotional and social loneliness. This study examined socio-demographic characteristics associated with emotional and social loneliness.

Methods: Data of 2251 community-dwelling older adults, included at the baseline measure of the Urban Health Centers Europe (UHCE) project, were analysed. Loneliness was measured with the 6-item De Jong-Gierveld Loneliness Scale. Multivariable logistic regression models were used to evaluate associations between age, sex, living situation, educational level, migration background, and loneliness.

Results: The mean age of participants was 79.7 years ( $S D=5.6$ years); $60.4 \%$ women. Emotional and social loneliness were reported by 29.2 and $26.7 \%$ of the participants; $13.6 \%$ experienced emotional and social loneliness simultaneously. Older age (OR: 1.16, 95\% Cl: 1.06-1.28), living without a partner (2.16, 95\% Cl: 1.73-2.70), and having a low educational level (OR: 1.82, 95\% Cl: 1.21-2.73), were associated with increased emotional loneliness. Women living with a partner were more prone to emotional loneliness than men living with a partner (OR: 1.78, 95\% Cl: 1.31-2.40). Older age (OR: 1.11, 95\% Cl: 1.00-1.22) and having a low educational level (OR: 1.77, 95\% Cl: 1.14-2.74) were associated with increased social loneliness. Men living without a partner were more prone to social loneliness than men living with a partner (OR: 1.94, 95\% Cl: 1.35-2.78).

Conclusions: Socio-demographic characteristics associated with emotional and social loneliness differed regarding sex and living situation. Researchers, policy makers, and healthcare professionals should be aware that emotional and social loneliness may affect older adults with different socio-demographic characteristics.
\end{abstract}

Keywords: Emotional loneliness, Social loneliness, Population characteristics

\section{Background}

Loneliness can be defined as an unpleasant experience, occurring when the quantity or quality of a person's social relationships is perceived to be deficient [1]. In general, feelings of loneliness motivate people to strengthen

\footnotetext{
* Correspondence: h.raat@erasmusmc.nl

'Department of Public Health, Erasmus University Medical Center, P.O. Box 2040, 3000, CA, Rotterdam, The Netherlands

Full list of author information is available at the end of the article
}

their existing social relationships or to build new relationships, after which these negative feelings may diminish [2]. However, for some people loneliness can become a chronic state. Persistent loneliness has been associated with negative outcomes for mental and physical health, such as depression, psychological distress, reduced selfesteem, cognitive impairment, functional decline, high blood pressure, cardiovascular diseases, and higher mortality rates $[2-7]$. 
Based on data collected in the third round (2006-07) of the European Social Survey, Yang and Victor [8] found that the prevalence of frequent loneliness among citizens aged 60 years and older, defined as feeling lonely 'most of the time' or 'all or almost all the time', varied between 19 and 34\% in Eastern Europe, 10-15\% in Southern Europe, and 3-9\% in Northern Europe. The prevalence of frequent loneliness was highest among adults aged 80 years and older [8]. Age-related changes and losses, such as deteriorating health, declining mobility, changing social roles, and the loss of a partner or friends have been associated with an increased susceptibility of loneliness in older age [4]. As European populations are ageing [9], loneliness can be expected to be a growing public health issue.

International studies provide an overview of sociodemographic characteristics associated with increased overall loneliness among older adults, such as widowhood, living in disadvantaged socioeconomic circumstances and having a migration background [4, 10-13].

However, few studies have distinguished between different dimensions of loneliness, such as emotional and social loneliness [3]. In 1973, Weiss [14] proposed that emotional loneliness is related to an absence of intimate attachments to other persons, whereas social loneliness is related to an absence of an engaging social network or a lack of social integration [14]. The onset of emotional loneliness may be related to the loss of intimate relationships, for example by a divorce or widowhood [14]. The onset of social loneliness may be related to the loss of a network of social relationships, for example by moving to another place [14]. Weiss [14] suggested that emotional loneliness may be characterized by feelings of isolation and anxiety, while social loneliness may be characterized by feelings of boredom, aimlessness, and marginality. Previous studies indicate that, despite being correlated, emotional and social loneliness can be recognized as distinct states affecting different groups of people [15-22].

The distinction between emotional and social loneliness may be relevant for the development of intervention strategies to reduce loneliness. According to the theoretical framework of Weiss, emotional loneliness may only be alleviated by a new or recovered intimate relationship, providing a sense of attachment, and social loneliness may only be alleviated by (re-)entering a social network, providing a sense of social integration [14, 21]. Many studies on the effects of intervention strategies did not report the impact on emotional and social loneliness [23]. In their meta-analysis, Masi, Chen [23] found that interventions to increase opportunities for social interaction or enhance social support had relatively small effects on reducing overall loneliness. Perissinotto, HoltLunstad [24] suggested that many interventions to reduce loneliness focus on the establishment of new social contacts, while this may only be beneficial for people who experience loneliness due to a lack of social contacts [24]. Interventions taking into account factors associated with the onset of loneliness, may be more appropriate [23]. Bouwman and Van Tilburg [25] have distinguished four intervention goals: 1) having a social network (related to social loneliness), 2) experiencing a sense of belonging (related to social loneliness), 3) experiencing meaning (related to emotional loneliness), and 4) experiencing intimacy (related to emotional loneliness). Intervention strategies may be used to reach multiple or specific intervention goals. For example, social skills training might be used to improve a person's social network; befriending interventions might be used to increase a sense of belonging; a voluntary job might be used to increase a sense of meaning [25].

In the current study, we examine which groups of older adults are at risk of emotional and social loneliness. We make a distinction between emotional and social loneliness because each dimension may require specific intervention strategies. Our study provides insight into the potential target groups for intervention strategies addressing emotional and/ or social dimensions of loneliness. The following research question is answered: Which socio-demographic characteristics are associated with emotional and social loneliness among older adults?

\section{Methods}

Data

Participants were 2325 community-dwelling older adults included in the baseline measure of the Urban Health Centres Europe (UHCE) project, a pre-post controlled trial to evaluate coordinated preventive care aiming to promote healthy ageing [26]. Multiple integrated care pathways (interventions) were implemented in the urban areas of Greater Manchester, The United Kingdom; Pallini, Greece; Rijeka, Croatia; Rotterdam, The Netherlands; Valencia, Spain. The UHCE study was approved by Medical Ethics Committees in all participating countries. UHCE was registered as ISRCTN52788952 [26]. Participants were recruited between May 2015 and June 2017. Eligible participants were (1) aged 75 years or older ( $\geq 70$ years in Greece and Spain), (2) living independently in selected neighbourhoods, (3) able to understand the local language, and (4) able to cognitively evaluate the risks and benefits of participation. All participants provided written informed consent. A full description of the inclusion process has been provided elsewhere [27]. The current study used baseline data collected by self-reported questionnaires. Participants with missing data of the De Jong-Gierveld Loneliness Scale $(n=35)$ or of socio-demographic characteristics $(n=39)$, 
were excluded. Hence, the population for analyses consisted of 2251 participants. Loneliness was measured by the 6-item De Jong-Gierveld Loneliness Scale [28] (Additional Table 1), a valid and reliable instrument to assess emotional and social loneliness among older adults living in diverse countries [29]. Response categories were 'no', 'more or less' and 'yes'. Scores were calculated according to the guidelines [28]. Scores varied between 0 'No loneliness' and 3 'Intense emotional or social loneliness'Scores $\geq 2$ indicating moderately intense or intense emotional loneliness were categorized as emotional and social loneliness [28]. The Loneliness Scale was originally developed in Dutch [30]. Validated translations were available in English and in Spanish. Forward-backward translation procedures were applied to translate the items from English into Croatian and Greek; the items were pilot tested among local older citizens. We used Cronbach's $\alpha$ to evaluate internal consistency of the 6item scale and 3-item sub scales for each country (Additional Table 2). The Cronbach's $\alpha$ for the 6 -item scale ranged between .62 (The United Kingdom) and .78 (The Netherlands). The Cronbach's $\alpha$ for the 3-item sub scale for emotional loneliness ranged between .56 (The United Kingdom) and .75 (The Netherlands). The Cronbach's $\alpha$ for the 3-item sub scale for social loneliness ranged between .68 (The United Kingdom) and .81 (Greece).

The following socio-demographic characteristics were studied: age (in years), sex (male/female), living situation (living with a partner/ living without a partner), educational level, and migration background. Educational level was divided into three categories, based on the International Standard Classification of Education (ISCED). ISCED level $0-1$ was categorized as 'primary or lower'; ISCED level 2-5 was categorized as 'secondary'; ISCED level 6-8 was categorized as 'tertiary' [31]. Migration background was assessed by country of birth. A country of birth other than the country of residence was categorized as a migration background. The country of residence was included as a covariate.

\section{Statistical analysis}

Descriptive statistics were used to characterize the participants. Multivariable logistic regression models were used to assess independent associations between socio-demographic characteristics and emotional, respectively, social loneliness. Odds ratios (ORs) and 95\% confidence intervals (95\% CI) were calculated for each factor. To assess moderating effects of sex, living situation and country for each factor, interaction terms were separately added to the multivariable models on emotional and social loneliness. Significant interaction effects were presented according to guidelines of Knol and VanderWeele [32].
A sensitivity analysis was conducted to examine whether associations between socio-demographic characteristics and loneliness differed when using a broader (cut-off value $\geq 1$ ) and a stricter definition (cut-off value =3) of loneliness (Additional Table 3). Data were analysed in Statistical Package for Social Sciences, version 25 for Windows (IBM SPSS Statistics for Windows, IBM Corp). $P$-values below .05 were considered to be statistically significant.

\section{Nonresponse analysis}

Characteristics of participants with missing data $(n=74)$ were compared with characteristics of participants with complete data $(n=2251)$ using chi-squared tests. Participants with missing data more often had a migration background $(P<.001)$, and more often lived with a partner $(P=.010)$ than participants without missing data. There were no differences regarding other sociodemographic characteristics.

\section{Results}

\section{Characteristics of the participants}

The socio-demographic characteristics of the participants are presented in Table 1. The mean age of participants was 79.7 years $(\mathrm{SD}=5.6$ years), $60.4 \%$ of the participants were women and $50.4 \%$ lived with a partner.

Emotional loneliness was reported by $29.2 \%(n=657)$ of the participants and social loneliness by $26.7 \%(n=$ $600)$ of the participants; $13.6 \%(n=306)$ experienced emotional and social loneliness simultaneously, $15.6 \%$ $(n=351)$ experienced emotional loneliness exclusively, and $13.1 \%(n=303)$ experienced social loneliness exclusively; $57.8 \%(n=1300)$ reported neither emotional or social loneliness. Women $(P<.001)$, participants living without a partner $(P<.001)$, and participants with a lower educational level $(P<.001)$ more often reported emotional loneliness. Participants living without a partner $(P=.002)$, participants with a lower educational level $(P=.006)$, and participants with a migration background $(P=.002)$ more often reported social loneliness.

\section{Associations between socio-demographic characteristics and emotional loneliness}

The multivariable model for emotional loneliness is presented in Table 2. Results of the interaction analysis for emotional loneliness are presented in Table 3. Significant interaction effects were found between sex and living situation $(P=.013)$, and between country and living situation $(P<.001)$. There were no other interaction effects for emotional loneliness $(p>.05)$. The multivariable model for emotional loneliness showed that older age (OR per 5 years: $1.16,95 \%$ CI: $1.06-1.28$ ) was associated with increased emotional loneliness. Older adults with a primary or lower educational level had 1.82 (95\% CI: 


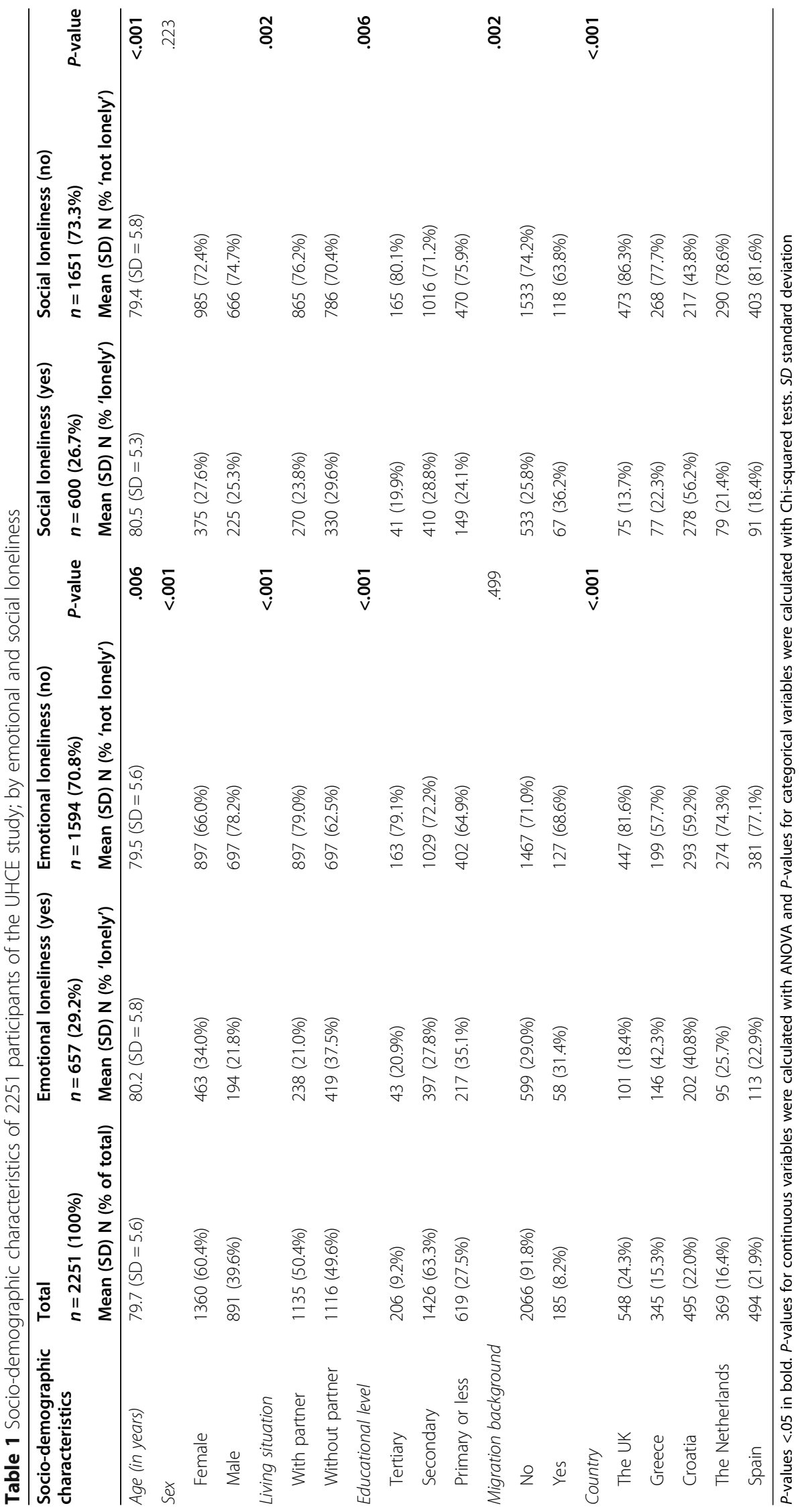


Table 2 Multivariable logistic regression models on associations between socio-demographic characteristics and emotional and social loneliness among 2251 participants of the UHCE study

\begin{tabular}{|c|c|c|}
\hline & Emotional loneliness ('yes' $n=657 ; 29.2 \%$ ) & Social loneliness ('yes' $n=600 ; 26.7 \%$ ) \\
\hline & Multivariable model OR $(95 \% \mathrm{Cl})$ & \\
\hline Age (per 5 years) & $1.16(1.06-1.28)$ & $1.11(1.00-1.22)$ \\
\hline Sex (female) & $1.38(1.10-1.72)$ & $0.86(0.69-1.09)$ \\
\hline Living situation (without partner) & $2.16(1.73-2.70)$ & $1.23(0.98-1.55)$ \\
\hline \multicolumn{3}{|l|}{ Educational level } \\
\hline Tertiary & ref. & ref. \\
\hline Secondary & $1.22(0.83-1.79)$ & $1.31(0.88-1.95)$ \\
\hline Primary or lower & $1.82(1.21-2.73)$ & $1.77(1.14-2.74)$ \\
\hline Migration background (yes) & $0.99(0.69-1.41)$ & $1.03(0.72-1.48)$ \\
\hline \multicolumn{3}{|l|}{ Country } \\
\hline The UK & ref. & ref. \\
\hline Greece & $4.33(2.96-6.34)$ & $1.88(1.24-2.85)$ \\
\hline Croatia & $3.11(2.32-4.16)$ & $8.34(6.14-11.33)$ \\
\hline The Netherlands & $1.50(1.07-2.09)$ & $1.66(1.16-2.37)$ \\
\hline Spain & $1.26(0.86-1.84)$ & $1.34(0.89-2.00)$ \\
\hline
\end{tabular}

Odds ratios and $95 \%$ confidence intervals are derived from multivariable logistic regression analyses for emotional and social loneliness (cut-off value Loneliness sub scales $\geq 2$ ). $P$-values $<.05$ in bold. $O R$ odds ratio, $C l$ confidence interval, ref. reference group

1.21-2.73) times higher odds of experiencing emotional loneliness compared to older adults with a tertiary educational level. Having a migration background was not associated with emotional loneliness.

Table 4 presents the odds ratios for emotional loneliness by sex and living situation. Women living with a partner had 1.78 (95\% CI: 1.31-2.40) times higher odds

Table 3 -values of the interaction terms added to the multivariable logistic regression models on associations between socio-demographic characteristics and emotional and social loneliness among 2251 participants of the UHCE study

\begin{tabular}{lll}
\hline Interaction terms & $\begin{array}{l}\text { Emotional } \\
\text { loneliness } \\
\boldsymbol{P} \text {-value }\end{array}$ & $\begin{array}{l}\text { Social } \\
\text { loneliness } \\
\boldsymbol{P} \text {-value }\end{array}$ \\
\hline Sex*age & .362 & .482 \\
Sex*country & .083 & .684 \\
Sex*living situation & .013 & .002 \\
Sex*educational level & .256 & .298 \\
Sex*migration background & .124 & .272 \\
Living situation*age & .562 & .693 \\
Living situation*country & $<.001$ & .693 \\
Living situation*educational level & .250 & .841 \\
Living situation*migration background $^{*}$ & .558 & .171 \\
Country*age & .322 & .854 \\
Country*educational level & .058 & .290 \\
Country*migration background & .907 & .620 \\
\hline
\end{tabular}

$P$-values were derived by separately adding the interaction terms to the multivariable logistic regression models for emotional and social loneliness (presented in Table 2). $P$-values $<.05$ in bold of experiencing emotional loneliness compared to men living with a partner. Living without a partner was associated with increased emotional loneliness among men and women. Men living without a partner had 3.10 (95\% CI: 2.17-4.40) times higher odds of experiencing emotional loneliness compared to men living with a partner. Women living without a partner had 1.79 (95\% CI: 1.37-2.33) times higher odds of experiencing emotional loneliness compared to women living with a partner.

Table 5 presents the odds ratios for emotional loneliness by country and living situation. In The United Kingdom (OR: 3.19, 95\% CI: 1.85-5.47), Greece (OR: 2.45, 95\% CI: 1.51-3.98), The Netherlands (OR: 5.31, 95\% CI: 3.00-9.40), and Spain (OR: 1.95, 95\% CI: $1.25-$ 3.02), living without a partner was associated with increased emotional loneliness. In Croatia, living without a partner was not associated with increased emotional loneliness (OR: 1.10; 95\% CI: .75-1.61).

\section{Associations between socio-demographic characteristics and social loneliness}

The multivariable model for social loneliness is presented in Table 2. Results of the interaction analysis for social loneliness are presented in Table 3. A significant interaction effect was found between sex and living situation $(P=.002)$. There were no other interaction effects for social loneliness $(p>.05)$.

The multivariable model for social loneliness showed older age was associated with increased social loneliness (OR per 5 years: 1.11, 95\% CI: 1.00-1.22), but nonsignificant $(P=.057)$. Older adults with a primary or 
Table 4 Results of analysis on interaction between sex and living situation for emotional loneliness among 2251 participants of the UHCE study

\begin{tabular}{|c|c|c|c|c|c|}
\hline \multicolumn{6}{|l|}{ Emotional loneliness } \\
\hline & \multicolumn{2}{|l|}{ Living with partner } & \multicolumn{2}{|l|}{ Living without partner } & \multirow{2}{*}{$\begin{array}{l}\text { OR }(95 \% \mathrm{Cl}) \text { for } \\
\text { living without } \\
\text { partner vs living } \\
\text { with partner withir } \\
\text { strata of sex }\end{array}$} \\
\hline & $\mathrm{N}$ lonely/not lonely & OR $(95 \% \mathrm{Cl})$ & $\mathrm{N}$ lonely/not lonely & OR $(95 \% \mathrm{Cl})$ & \\
\hline Male & $113 / 546$ & ref. & $81 / 151$ & $3.10(2.17-4.40)$ & $3.10(2.17-4.40)$ \\
\hline Female & $125 / 351$ & $1.78(1.31-2.40)$ & $338 / 546$ & $3.17(2.45-4.12)$ & $1.79(1.37-2.33)$ \\
\hline $\begin{array}{l}\text { OR }(95 \% \mathrm{Cl}) \text { for female vs male } \\
\text { within strata of living situation }\end{array}$ & & $1.78(1.31-2.40)$ & & $1.02(0.74-1.41)$ & \\
\hline
\end{tabular}

Interaction between sex and living situation on the multiplicative scale $P=.013$. ORs were presented for each stratum with male living with a partner as the reference group. ORs for sex were presented within strata of living situation; ORs for living situation were presented within strata of sex. ORs are adjusted for age, educational level, migration background and country. $P$-values $<.05$ in bold. OR odds ratio, $C l$ confidence interval, ref. reference group

lower educational level had 1.77 (95\% CI: 1.14-2.74) times higher odds of experiencing social loneliness compared to older adults with a tertiary educational level. Having a migration background was not associated with social loneliness.

Table 6 presents the odds ratios for social loneliness by sex and living situation. Men living without a partner had 1.94 (95\% CI: 1.35-2.78) times higher odds of experiencing social loneliness compared to men living with a partner. Among women, living without a partner was not associated with increased social loneliness.

\section{Simultaneous emotional and social loneliness}

In the study population, some participants only reported the presence of one type of loneliness, while others reported the presence of both types of loneliness. To explore whether the associations were different when only one type of loneliness was reported, or when both types of loneliness were reported, we performed additional analyses in which we compared these groups with older adults experiencing neither emotional or social loneliness (Additional Tables 4 and 5). The directions of the associations for exclusive emotional loneliness and exclusive social loneliness were similar. For the simultaneous experience of emotional and social loneliness, the results were similar to the results of emotional loneliness.

\section{Sensitivity analysis}

The multivariable models using a broader and a stricter definition of loneliness are presented in Additional Table 3. The directions of the associations between sociodemographic characteristics and emotional and social loneliness were similar when using $\geq 1, \geq 2$, and $=3$ as cut-off scores for the Loneliness sub scales.

\section{Discussion and conclusions}

Older age, living without a partner, and having a low educational level were independently associated with increased emotional loneliness among older adults. Women living with a partner were more prone to emotional loneliness than men living with a partner. Older age and having a low educational level were associated with increased social loneliness. Men living without a partner were more prone to social loneliness than men living with a partner. With regard to the simultaneous

Table 5 Results of analysis on interaction between country and living situation for emotional loneliness among 2251 participants of the UHCE study

\begin{tabular}{|c|c|c|c|c|c|}
\hline \multicolumn{6}{|c|}{ Emotional loneliness } \\
\hline & \multicolumn{2}{|l|}{ Living with partner } & \multicolumn{2}{|l|}{ Living without partner } & \multirow{2}{*}{$\begin{array}{l}\text { OR }(95 \% \mathrm{Cl}) \text { for } \\
\text { living without } \\
\text { partner vs living } \\
\text { with partner within } \\
\text { strata of country }\end{array}$} \\
\hline & $N$ lonely/not lonely & OR $(95 \% \mathrm{Cl})$ & $N$ lonely/not lonely & OR $(95 \% \mathrm{Cl})$ & \\
\hline The UK & $19 / 210$ & ref. & $82 / 237$ & $3.19(1.85-5.47)$ & $3.19(1.85-5.47)$ \\
\hline Greece & $81 / 157$ & $5.53(3.10-9.84)$ & $65 / 42$ & $13.56(7.11-25.87)$ & $2.45(1.51-3.98)$ \\
\hline Croatia & $75 / 130$ & $6.31(3.63-10.95)$ & $127 / 163$ & $6.93(4.05-11.85)$ & $1.10(0.75-1.61)$ \\
\hline The Netherlands & $19 / 171$ & $1.11(0.56-2.17)$ & $76 / 103$ & $5.87(3.31-10.41)$ & $5.31(3.00-9.40)$ \\
\hline Spain & $44 / 229$ & $1.78(0.97-3.28)$ & $69 / 152$ & $3.46(1.91-6.28)$ & $1.95(1.25-3.02)$ \\
\hline
\end{tabular}

Interaction between country and living situation on the multiplicative scale $P<.001$

ORs are adjusted for age, sex, educational level and migration background. $P$-values $<.05$ in bold. OR odds ratio, $C l$ confidence interval, ref. reference group 
Table 6 Results of analysis on interaction between sex and living situation for social loneliness among 2251 participants of the UHCE study

\begin{tabular}{|c|c|c|c|c|c|}
\hline \multicolumn{6}{|l|}{ Social loneliness } \\
\hline & \multicolumn{2}{|l|}{ Living with partner } & \multicolumn{2}{|l|}{ Living without partner } & \multirow{2}{*}{$\begin{array}{l}\text { OR }(95 \% \mathrm{Cl}) \text { for } \\
\text { living without } \\
\text { partner vs } \\
\text { living with } \\
\text { partner within } \\
\text { strata of sex }\end{array}$} \\
\hline & $N$ lonely/not lonely & OR $(95 \% \mathrm{Cl})$ & $N$ lonely/not lonely & OR $(95 \% \mathrm{Cl})$ & \\
\hline Male & $148 / 511$ & ref. & $77 / 155$ & $1.94(1.35-2.78)$ & $1.94(1.35-2.78)$ \\
\hline Female & $122 / 354$ & $1.18(0.87-1.59)$ & $253 / 631$ & $1.11(0.86-1.43)$ & $0.94(0.71-1.25)$ \\
\hline $\begin{array}{l}\text { OR }(95 \% \mathrm{Cl}) \text { for female vs } \\
\text { male within strata of living situation }\end{array}$ & & $1.18(0.87-1.59)$ & & $0.57(0.41-0.80)$ & \\
\hline
\end{tabular}

male within strata of living situation

Interaction between sex and living situation on the multiplicative scale $P=.002$

ORs are adjusted for age, educational level, migration background and country. $P$-values $<.05$ in bold. OR odds ratio, $C l$ confidence interval, ref. reference group

experience of emotional and social loneliness, the results were similar to the results of emotional loneliness.

In our study among participants with a mean age of almost 80 years, older age was associated with increased emotional loneliness and with increased social loneliness (borderline significance). Results of a meta-analysis [10] showed that older age was associated with increased overall loneliness in studies among participants with a mean age $>80$ years, but not among participants with a mean age of $60-80$ years. Further research could examine possible differences in age-related factors associated with emotional and social loneliness, and their onset. The death of a partner may primarily be associated with emotional loneliness, whereas leaving paid employment and decreasing out-door mobility could be age-related factors primarily associated with social loneliness [33].

Women living with a partner were more prone to emotional loneliness than men living with a partner. This is in line with results of previous studies [34, 35]. Dykstra and de Jong Gierveld [35] have suggested that married men might more often rely on their partner for emotional support than married women, and tend to experience more emotional fulfilment in marriage [34, 35]. Distinguishing between emotional and social loneliness in future studies, and testing for interactions between sex and living situation or marital status, may clarify the association between sex and loneliness [10].

Living without a partner was associated with increased emotional loneliness, which is in line with results of previous studies $[15,18]$. In addition, living without a partner was associated with increased social loneliness among men. This corresponds to findings of Dykstra and Fokkema [34], as results of their study showed that divorced men had a greater vulnerability to social loneliness than divorced women. Dykstra and Fokkema [34] have suggested that married women might more often be in charge of social activities, have bigger and more varied social networks and are less likely to lose social contacts after a divorce [34, 35].
In Croatia, living without a partner was not associated with increased emotional loneliness. The association between living situation and loneliness may be influenced by cultural norms and values, affecting individual expectations of family members [36]. Further research is needed to explain cross-country differences in the association between living situation and emotional loneliness. Descriptive statistics on emotional loneliness distinguishing between participants living with/ without children have been provided in Additional Table 6 .

In general, data used in this study indicate that the proportion of older persons experiencing exclusive or combined emotional and social loneliness varies between European countries (Additional Table 4). Hansen and Slagsvold [35] suggest cross-country differences in the risk of late-life loneliness can be explained by macrolevel inequalities in health, socioeconomic status, marital status, and social integration. In addition, cross-country differences may be explained by differences in social welfare, demographic composition, and cultural norms and values [36]. Having a low educational level was associated with increased emotional and social loneliness, which is in line with results of previous studies [4, 10, 15, 37]. Older adults with a low educational level are more likely to live in disadvantaged socioeconomic circumstances, which have been associated with chronic stress and a decreased quality of social relations [38]. In addition, living in disadvantaged socioeconomic circumstances has been associated with reduced opportunities for participation in social activities [4].

Having a migration background was not associated with loneliness, in contrast to results of previous studies [39, 40]. Older adults with a migration background might be at increased risk of loneliness as a result of language barriers, cultural differences, possible encounters with discrimination and racism, and a dislocation of social networks after transnational migration [39, 40]. In our study, social loneliness was reported more frequently by participants with a migration background, but there 
was no independent association. This may have differed between immigrant groups, depending on their command of the local language and the magnitude of cultural differences [39]. However, in our study the number of participants with a migration background was too low to distinguish between groups.

\section{Methodological considerations}

A strength of this study was the relatively high average age of the study population. Older adults living in urban areas in Southern, Western and Eastern European countries were represented in the sample, which has increased the external validity of the results. Using a broader and a stricter definition of loneliness yielded similar results, indicating that the findings are applicable to older adults experiencing loneliness at different intensities.

Nevertheless, several limitations need to be considered when interpreting the findings. First, a sampling bias cannot be ruled out. Older adults with poor health may have been less likely to participate in the UHCE study [27], participants living with a partner and participants with a migration background were more often excluded from the population for analyses due to missing data. This has reduced the representativeness of the sample, and should be considered when the findings are generalized. Secondly, although previous studies reported good psychometric properties of the 6-item De Jong-Gierveld Loneliness Scale among culturally diverse groups, in our study, the internal consistency of the emotional loneliness sub scale was relatively low in the United Kingdom and Greece. We therefore recommend to consider the use of the 11-item De Jong Gierveld Loneliness scale in future studies distinguishing between emotional and social loneliness $[28,30]$.

Thirdly, the number of participants with a migration background was relatively low, and several sub groups in the interaction analyses were small, which may have resulted in a lack of statistical power to evaluate differences. In the multivariable logistic regression models, some $95 \%$ confidence intervals were relatively large. Future studies should expand upon the findings using longitudinal designs with large and varied samples of older adults. Lastly, causal directions of the associations between socio-demographic characteristics and loneliness could not be examined. Longitudinal research is needed to evaluate (bi-)directional associations between living situation, educational level, migration background and loneliness.

\section{Implications for policy and practice}

Our findings may be used to identify target groups for intervention strategies aimed at the emotional or social dimension of loneliness. As mentioned above, Bouwman and Van Tilburg [25] have distinguished four intervention goals. Based on the results of this study, we hypothesize that intervention strategies aiming 1) to build or to strengthen a social network, and intervention strategies aiming 2) to increase a sense of belonging could in particular be beneficial for older adults, for men living without a partner, and for older adults with a low educational level. Intervention strategies aiming 3) to increase a sense of meaning, and intervention strategies aiming 4) to increase intimacy in relationships could in particular be beneficial for older adults of higher age, for older adults living without a partner, for women living with a partner, and for older adults with a low educational level. Future studies need to evaluate which intervention strategies are most effective in reducing emotional and/ or social loneliness [22].

\section{Conclusions}

Older adults of higher age, women living with a partner, older adults living without a partner, and older adults with a low educational level may be at increased risk of emotional loneliness. Older adults of higher age, men living without a partner, and older adults with a low educational level may be at increased risk of social loneliness. More research in diverse populations, using longitudinal designs, is needed to confirm these findings. In the meantime, healthcare professionals and policy makers are advised to pay attention to an increased susceptibility of emotional and social loneliness in the above mentioned sub groups. We recommend to further develop effective and feasible interventions to prevent and alleviate specific dimensions of loneliness among older adults to contribute to their health and wellbeing.

\section{Supplementary Information}

The online version contains supplementary material available at https://doi. org/10.1186/s12877-021-02058-4.

Additional file1: Supplementary tables Socio-demographic factors associated with emotional and social loneliness among older adults.

\section{Abbreviations}

UHCE: Urban Health Centers Europe; ISCED: International Standard Classification of Education; OR: Odds ratio; B: Beta; $95 \%$ Cl: $95 \%$ confidence interval; a: Alpha

\section{Acknowledgements}

Not applicable.

\section{Authors' contributions}

The study was designed by IF, ST and HR. IF, ST and HR analysed and interpreted the data. IF drafted the manuscript. ST was a major contributor in writing the manuscript. GW, TA, EK, LB, AV, AM, FMR, W, CF and HR have contributed to the design and the data collection of the Urban Health Centers Europe study and have revised the current manuscript. All authors have read and approved the final version of this manuscript.

\section{Funding}

This work was supported by the European Union, CHAFEA, third health programme, number 20131201. The funding body played no role in the 
design of the study, the collection, analysis, and interpretation of data, or in writing the manuscript.

\section{Availability of data and materials}

The datasets used and/or analysed during the current study are available from the corresponding author on reasonable request.

\section{Ethics approval and consent to participate}

Ethical committee procedures have been followed in all cities and institutions involved, and approval has been provided. The names of the review board and the approval references are: Manchester, United Kingdom: NRES Committee West Midlands - Coventry \& Warwickshire; 06-03-2015; 15/ WM/0080; NRES Committee South Central - Berkshire B; 29-20-2014; 14/SC/ 1349; Pallini, Greece: The Ethics and Scientific board - Latriko Palaiou Falirou Hospital; 04/03/2015; 20150304-01; Rijeka, Croatia: The Ethical Committee Faculty of Medicine University of Rijeka; 07-04-2014; 2170-24-01-14-02; Rotterdam, The Netherlands: Medische Ethische Toetsings Commissie (METC) Erasmus MC Rotterdam; 08/01/2015; MEC-2014-661; Valencia, Spain: Comisión de Investigación - Consorcio Hospital General Universitario de Valencia. 29/ 01/2015; CICHGUV-2015-01-29. Written consent is obtained from all participants. The data used in this study were anonymized before its use.

\section{Consent for publication}

Not applicable.

\section{Competing interests}

The authors declare that they have no competing interests.

\section{Author details}

'Department of Public Health, Erasmus University Medical Center, P.O. Box 2040, 3000, CA, Rotterdam, The Netherlands. ${ }^{2}$ Manchester Urban Collaboration on Health, Centre for Epidemiology, Division of Population Health, Health Services Research and Primary Care, Manchester Academic Health Science Centre, The University of Manchester, Manchester, UK. ${ }^{3}$ Polibienestar Research Institute, University of Valencia, Valencia, Spain. ${ }^{4}$ Research Centre Innovation in Care, Rotterdam University of Applied Sciences Rotterdam, Rotterdam, The Netherlands. ${ }^{5}$ Department of Social Medicine and Epidemiology, Faculty of Medicine, University of Rijeka, Rijeka, Croatia. ${ }^{6}$ Alliance for Integrated Care, Athens, Greece. ${ }^{7}$ Division of Geriatrics, Department of Internal Medicine, Erasmus MC University Medical Center, Rotterdam, The Netherlands.

Received: 14 April 2020 Accepted: 1 February 2021

\section{Published online: 09 February 2021}

\section{References}

1. Perlman D, Peplau LA. Toward a social psychology of loneliness. Pers Relat. 1981;3:31-56.

2. Hawkley LC, Cacioppo JT. Loneliness matters: a theoretical and empirical review of consequences and mechanisms. Ann Behav Med. 2010;40(2):21827.

3. Courtin E, Knapp M. Social isolation, loneliness and health in old age: a scoping review. Health Soc Care Commun. 2017;25(3):799-812.

4. Cohen-Mansfield J, Hazan H, Lerman Y, Shalom V. Correlates and predictors of loneliness in older-adults: a review of quantitative results informed by qualitative insights. Int Psychogeriatr. 2016;28(4):557-76.

5. Luanaigh CÓ, Lawlor BA. Loneliness and the health of older people. Int J Geriatr Psychiatr. 2008:23(12):1213-21.

6. Valtorta NK, Kanaan M, Gilbody S, Ronzi S, Hanratty B. Loneliness and social isolation as risk factors for coronary heart disease and stroke: systematic review and meta-analysis of longitudinal observational studies. Heart. 2016; 102(13):1009-16.

7. Holt-Lunstad J, Smith TB, Baker M, Harris T, Stephenson D. Loneliness and social isolation as risk factors for mortality: a meta-analytic review. Perspect Psychol Sci. 2015;10(2):227-37.

8. Yang K, Victor C. Age and loneliness in 25 European nations. Ageing Soc. 2011;31(8):1368-88.

9. Eurostat. Population structure and ageing: European Union, 1995-2013 2014 [Available from: http://ec.europa.eu/eurostat/statistics-explained/index.php/ Population_structure_and_ageing. Accessed 15/10/2020.
10. Pinquart $M$, Sorensen S. Influences on loneliness in older adults: a metaanalysis. Basic Appl Soc Psychol. 2001;23(4):245-66.

11. de Jong Gierveld J, van Tilburg T, Dykstra P. Loneliness and social isolation: new ways of theorizing and conducting research. 2018.

12. Victor CR, Scambler SJ, Bowling ANN, Bond J. The prevalence of, and risk factors for, loneliness in later life: a survey of older people in Great Britain. Ageing Soc. 2005;25(6):357-75.

13. Aartsen $M$, Jylhä M. Onset of loneliness in older adults: results of a 28 year prospective study. Eur J Ageing. 2011;8(1):31-8.

14. Weiss RS. Loneliness: the experience of emotional and social isolation. 1973

15. Dahlberg L, McKee KJ. Correlates of social and emotional loneliness in older people: evidence from an English community study. Aging Ment Health. 2014:18(4):504-14.

16. DiTommaso E, Spinner B. The development and initial validation of the social and emotional loneliness scale for adults (SELSA). Personal Individ Differ. 1993;14(1):127-34.

17. DiTommaso E, Spinner B. Social and emotional loneliness: a re-examination of Weiss' typology of loneliness. Personal Individ Differ. 1997;22(3):417-27.

18. Drennan J, Treacy M, Butler M, Byrne A, Fealy G, Frazer K, et al. The experience of social and emotional loneliness among older people in Ireland. Ageing Soc. 2008;28(8):1113-32.

19. Green LR, Richardson DS, Lago T, Schatten-Jones EC. Network correlates of social and emotional loneliness in young and older adults. Personal Soc Psychol Bull. 2001;27(3):281-8.

20. Hawkley LC, Browne MW, Cacioppo JT. How can I connect with thee? Let me count the ways. Psychol Sci. 2005;16(10):798-804.

21. Russell D, Cutrona CE, Rose J, Yurko K. Social and emotional loneliness: an examination of Weiss's typology of loneliness. J Pers Soc Psychol. 1984;46(6): 1313.

22. Schnittger RIB, Wherton J, Prendergast D, Lawlor BA. Risk factors and mediating pathways of loneliness and social support in communitydwelling older adults. Aging Ment Health. 2012;16(3):335-46.

23. Masi CM, Chen H-Y, Hawkley LC, Cacioppo JT. A meta-analysis of interventions to reduce loneliness. Personal Soc Psychol Rev. 2011;15(3):219-66.

24. Perissinotto C, Holt-Lunstad J, Periyakoil VS, Covinsky K. A practical approach to assessing and mitigating loneliness and isolation in older adults. J Am Geriatr Soc. 2019;67(4):657-62.

25. Bouwman TE, Van Tilburg TG. Towards a more focused approach to Ioneliness: Seven active elements in loneliness interventions. Tijdschrift Voor Gerontol Geriatr. 2020:51:1

26. Franse CB, Voorham AJJ, Van Staveren R, Koppelaar E, Martijn R, ValíaCotanda $\mathrm{E}$, et al. Evaluation design of urban health Centres Europe (UHCE): preventive integrated health and social care for community-dwelling older persons in five European cities. BMC Geriatr. 2017;17(1):209.

27. Franse $C B$, van Grieken $A$, Alhambra-Borrás T, Valía-Cotanda E, van Staveren $R$, Rentoumis $T$, et al. The effectiveness of a coordinated preventive care approach for healthy ageing (UHCE) among older persons in five European cities: a pre-post controlled trial. Int J Nurs Stud. 2018;88:153-62.

28. Gierveld JDJ, Tilburg TV. A 6-item scale for overall, emotional, and social Ioneliness: confirmatory tests on survey data. Res Aging. 2006;28(5):582-98.

29. Gierveld JDJ, Van Tilburg T. The De Jong Gierveld short scales for emotiona and social loneliness: tested on data from 7 countries in the UN generations and gender surveys. Eur J Ageing. 2010:7(2):121-30.

30. De Jong-Gierveld J, Kamphuls F. The development of a Rasch-type Ioneliness scale. Appl Psychol Meas. 1985:9(3):289-99.

31. Statistics UIf. International standard classification of education: ISCED 2011: UNESCO Institute for Statistics Montreal; 2012.

32. Knol MJ, VanderWeele TJ. Recommendations for presenting analyses of effect modification and interaction. Int J Epidemiol. 2012;41(2):514-20.

33. Cohen-Mansfield J, Parpura-Gill A. Loneliness in older persons: a theoretical model and empirical findings. Int Psychogeriatr. 2007;19(2):279-94.

34. Dykstra PA, Fokkema T. Social and emotional loneliness among divorced and married men and women: comparing the deficit and cognitive perspectives. Basic Appl Soc Psychol. 2007:29:1-12

35. Dykstra PA, de Jong Gierveld J. Gender and marital-history differences in emotional and social loneliness among Dutch older adults. Can J Aging. 2004;23(2):141-55.

36. de Jong GJ, Tesch-Römer C. Loneliness in old age in eastern and Western European societies: theoretical perspectives. Eur J Ageing. 2012;9(4):285-95.

37. Hansen T, Slagsvold B. Late-life loneliness in 11 European countries: results from the generations and gender survey. Soc Indic Res. 2016;129(1):445-64. 
38. Hawkley LC, Hughes ME, Waite LJ, Masi CM, Thisted RA, Cacioppo JT. From social structural factors to perceptions of relationship quality and loneliness: the Chicago health, aging, and social relations study. J Gerontol Ser B Psychol Sci Soc Sci. 2008;63(6):S375-S84.

39. Gierveld JDJ, Van der Pas S, Keating N. Loneliness of older immigrant groups in Canada: effects of ethnic-cultural background. J Cross-Cult Gerontol. 2015;30(3):251-68.

40. Victor $C R$, Burholt $V$, Martin W. Loneliness and ethnic minority elders in Great Britain: an exploratory study. J Cross-Cult Gerontol. 2012;27(1):65-78.

\section{Publisher's Note}

Springer Nature remains neutral with regard to jurisdictional claims in published maps and institutional affiliations.

Ready to submit your research? Choose BMC and benefit from:

- fast, convenient online submission

- thorough peer review by experienced researchers in your field

- rapid publication on acceptance

- support for research data, including large and complex data types

- gold Open Access which fosters wider collaboration and increased citations

- maximum visibility for your research: over $100 \mathrm{M}$ website views per year

At BMC, research is always in progress.

Learn more biomedcentral.com/submissions 